\title{
FORCED DIFFERENCES BETWEEN TERMS OF SUBSEQUENCES OF INTEGER SEQUENCES
}

\author{
MICHAEL GILPIN AND ROBERT SHELTON
}

\begin{abstract}
Let $a_{1}, a_{2} \ldots$ be a sequence of integers and let $D=\left\{d_{1} \ldots d_{h}\right\}$ be a fixed finite set of integers. For each positive integer $n$ we investigate the problem of choosing maximal subsequences $a_{i_{1}} \ldots, a_{i_{f}}$ from $a_{1} \ldots \ldots a_{n}$ such that $\left|a_{i_{\alpha}}-a_{i_{\beta}}\right| \notin D$ for $\alpha \neq \beta$. An asymptotic form for $t$, the maximum length of such subsequences, is derived in the special case $a_{1}=i$.
\end{abstract}

0. Introduction. We examine the problem of constructing subsequences $a_{i_{1}}, \ldots, a_{i_{1}}$ of a finite sequence $a_{1}, \ldots, a_{n}$ of integers with the property that the differences $\left|a_{i_{\alpha}}-a_{i_{\beta}}\right|$ avoid a fixed set $D$ of integers. The problem is illustrated by three examples in $\S 1$. In $\$ 2$ a partial solution of the problem is stated and proved using the pigeonhole principle. In $\S 3$ we define, and give some elementary properties of the function $L\left(\left\{a_{i}\right\}_{1}^{\infty}, D: n\right)$, where $L\left(\left\{a_{i}\right\}_{1}^{\infty}, D: n\right)$ is the length of the longest subsequence $a_{i_{1}}, \ldots, a_{i_{t}}$ that can be chosen from $a_{1}, \ldots, a_{n}$ so that the differences $\left|a_{i_{\alpha}}-a_{i_{\beta}}\right|$ are never in $D$. In $\$ 4$ we prove that for the sequence $1,2,3, \ldots$ of positive integers, the function $L$ is essentially cyclic, and we investigate its asymptotic behavior.

1. Three example problems. Consider the following three problems, each of which concerns choosing subsequences of an increasing sequence of integers such that the differences between the terms of the subsequence either avoid or lie in a fixed set of integers.

Problem One (Liu [2 or 3]). "A chess player wants to prepare for a championship match by playing some practice games in 77 days. He wants to play at least one match a day but no more than 132 games altogether. Show that no matter how he schedules the games there is a period of consecutive days within which he plays exactly 21 games".

Problem Two (Gilpin and Shelton [1]). "An elevator starts at the top floor of a one hundred story building and makes a total of 40 stops on its way down to the first floor. (Both the top and bottom floors count as stops.) Show that somewhere in its travel the elevator had to stop at two floors that were exactly 9,10 or 19 stories apart”.

Problem Three. Can the first two problems be improved? I.e., would the conclusion in Problem One still hold if 132 games were played in a span of less than 77 days? Would the conclusion of Problem Two still be valid if the elevator made fewer than 40 stops?

Received by the editors July 26, 1982.

1980 Mathematics Subject Classification. Primary 10L10.

Key words and phrases. Subsequences, blocks, pigeonhole principle.

(C)1983 American Mathematical Society $0002-9939 / 82 / 0000-1216 / \$ 03.25$ 
Similar problems of this type easily come to mind. For example, what is the length of the longest subsequence of $1,2, \ldots, 100$ whose terms never differ by a prime? (Whose terms never differ by a multiple of 5?)

2. A solution to Problems One and Two. We state and prove a general result.

THEOREM 2.1. Let $1 \leqslant a_{1}<a_{2}<\cdots<a_{n}$ and $0=d_{0}<d_{1}<\cdots<d_{k}$ be increasing sequences of integers. If $(k+1) \cdot n>a_{n}+d_{k}$, then there exist integers $i, j, l, m$ such that $i \neq j, l \neq m$ and $\left|a_{i}-a_{j}\right|=\left|d_{l}-d_{m}\right|$.

Proof. Our proof is patterned after Liu's solution (see [2 or 3]) to Problem One. Consider the inequalities

$$
\begin{gathered}
1+d_{0} \leqslant a_{1}+d_{0}<a_{2}+d_{0}<\cdots<a_{n}+d_{0} \\
\vdots \\
1+d_{k} \leqslant a_{1}+d_{k}<a_{2}+d_{k}<\cdots<a_{n}+d_{k} .
\end{gathered}
$$

These inequalities involve a total of $(k+1) \cdot n$ integers of the form $a_{i}+d_{j}$. Since each of these integers lies in the interval $1, \ldots, a_{n}+d_{k}$, the pigeonhole principle implies at least two of these, say $a_{i}+d_{m}$ and $a_{j}+d_{l}$ must be equal. The theorem follows immediately from this observation.

Problem One can now be seen to follow from Theorem 2.1 by taking $d_{1}=21$ and letting $a_{i}$ denote the total number of games played through the $i$ th day. Since $2 \cdot 77>132+21=153$, the hypothesis of the theorem is satisifed. Problem Two also follows from Theorem 2.1 by taking $d_{1}=9$ and $d_{2}=19$. One need only check that $3 \cdot 40>100+19=119$. Note that in this case the values of $\left|d_{l}-d_{m}\right|$ for $l \neq m$ are 9,10 and 19 .

The proof of Theorem 2.1 and, in particular, Liu's elegant solution to the chess player problem, are deceptive in that these proofs give the impression that the conclusions are the best possible. Thus, in Liu's solution (see [2 or 3]) to Problem One, one has a total of 154 integers of the form $a_{i}+0$ or $a_{i}+21$ in the interval $[1,153]$ which would seem to be the minimal hypothesis from which to obtain $a_{i}=a_{j}+21$ for terms $a_{i}$ and $a_{j}$. However, when the authors wrote a PASCAL program to investigate problems like Problem One and Problem Two and then applied this program to Liu's problem (Problem One), the program showed the conclusion of the chess player problem still obtained for sequences of 76 days, of 75 days, ..., even for sequences of 70 days.

Note that finding the minimal number of days in the chess player problem, so that one can still conclude a sequence of consecutive days occurs during which exactly 21 games were played, is equivalent to finding the maximal subsequence of $1,2, \ldots, 132$ whose terms never differ by 21 . All seventy-term subsequences of $1,2, \ldots, 132$ contain at least two terms that differ by 21 , and 70 is minimal with respect to this property, a contention we will show in $\$ 3$. Equivalently, the longest subsequence of $1,2, \ldots, 132$ whose terms never differ by 21 has length sixty-nine. In the remaining sections of this paper we examine problems of this sort from this alternate point of view, i.e., we search for maximal subsequences, the differences of whose terms avoid a fixed set of integers. 
3. The length function $L$. We carefully define a function $L: Z^{+} \rightarrow Z^{+}$and use this function to investigate problems of the type posed in Problem Three.

Definition. Let $a_{1}, a_{2}, \ldots$ be an increasing sequence of integers and let $D=$ $\left\{d_{1}, d_{2}, \ldots, d_{k}\right\}$ be a (finite) nonvoid set of positive integers. Then for each positive integer $n, L\left(\left\{a_{i}\right\}_{1}^{\infty}, D: n\right)$ is defined to be the length of the longest subsequence $a_{i_{1}}, a_{i_{2}}, \ldots, a_{i_{1}}$ of $a_{1}, a_{2}, \ldots, a_{n}$ such that $\alpha \neq \beta$ implies $\left|a_{i_{\alpha}}-a_{i_{\beta}}\right| \notin D$.

In other words, $L$ gives the length of the longest subsequence, the differences of whose terms avoid a fixed set $D$ of integers. Three elementary properties of this function are given in

THEOREM 3.1. Let $\left\{a_{i}\right\}_{1}^{\infty}$ be an increasing sequence of integers, and let $D$ and $D^{\prime}$ be nonvoid sets of positive integers. Then for all positive integers $n$ and $m$ :

(i) $D^{\prime} \supset D$ implies $L\left(\left\{a_{i}\right\}_{1}^{\infty}, D^{\prime}: n\right) \leqslant L\left(\left\{a_{i}\right\}_{1}^{\infty}, D: n\right)$;

(ii) $m>n$ implies $L\left(\left\{a_{i}\right\}_{1}^{\infty}, D: m\right) \geqslant L\left(\left\{a_{i}\right\}_{1}^{\infty}, D: n\right)$; and .

(iii) $L\left(\left\{a_{i}\right\}_{1}^{\infty}, D: m+n\right) \leqslant m+L\left(\left\{a_{i}\right\}_{1}^{\infty}, D: n\right)$.

The proof of Theorem 3.1 is immediate from the definition of $L$.

For the majority of this paper we restrict our attention to the case where $\left\{a_{i}\right\}_{1}^{\infty}$ is the sequence of positive integers. For brevity we write $L(\{1,2, \ldots\}, D: n)$ as $L(D: n)$. Our next result shows $L(D: n)$ is subadditive with respect to its integer argument.

THEOREM 3.2. Let $D$ be any nonvoid set of positive integers. Then for all positive integers $n$ and $m$,

$$
L(D: n+m) \leqslant L(D: n)+L(D: m)
$$

Proof. Let $a_{1}<\cdots<a_{t}$ be a subsequence of $1,2, \ldots, n+m$ with $t=$ $L(D: n+m)$. Suppose $a_{k}$ is the largest term of this subsequence which is less than or equal to $n$. Then $a_{1}, \ldots, a_{k}$ is a subsequence of $1, \ldots, n$ with $\left|a_{i}-a_{j}\right| \notin D$ whenever $i \neq j$. Likewise, $a_{k+1}-n, \ldots, a_{t}-n$ is a subsequence of $1, \ldots, m$ such that $i \neq j$ implies $\left|\left(a_{i}-n\right)-\left(a_{j}-n\right)\right| \notin D$. Thus $L(D: n) \geqslant k$ and $L(D: m) \geqslant t-k$. This establishes Theorem 3.2.

For arithmetic progressions $L$ is always subadditive with respect to its integer argument. However, in general, subadditivity depends on the choice of $D$. For example if $\left\{a_{i}\right\}_{1}^{\infty}$ is the sequence $a_{1}=1, a_{i+1}=2 i$ for $i \geqslant 1$, then subadditivity holds if $D=\{2\}$ and fails if $D=\{1\}$.

In special cases one obtains formulas for $L(D: n)$ in closed form.

THEOREM 3.3. If $D=\{d, 2 \cdot d, \ldots, k \cdot d\}$ is the set of the first $k$ multiples of a fixed integer $d$, then

$$
L(D: n)=d \cdot \llbracket \frac{n}{(k+1) \cdot d} \rrbracket+\min \{d, n \bmod [(k+1) \cdot d]\},
$$

where the brackets denote the greatest integer function. 
Proof. We first show $L(D:(k+1) d)=d$. Let $a_{1}<\cdots<a_{t}$ be any subsequence of $1,2, \ldots,(k+1) d$ with the property that $i \neq j$ implies $\left|a_{i}-a_{j}\right| \neq m d$ for $m=$ $1, \ldots, k$. Note that $a_{i} \equiv a_{j}(\bmod d)$ forces $\left|a_{i}-a_{j}\right|=m d$ for some $m$ with $1 \leqslant m \leqslant k$. Therefore each of the $t$ terms of the subsequence $a_{1}, \ldots, a_{t}$ has a distinct least nonnegative remainder upon division by $d$. Thus $t \leqslant d$, so $L(D:(k+1) d) \leqslant d$.

We can show $L(D:(k+1) d) \geqslant d$ by simply taking $a_{i}=i$ for $i=1, \ldots, d$. Thus $L(D:(k+1) d)=d$. If $n<(k+1) d$, much the same argument shows $L(D: n)=$ $\min \{d, n\}$.

Finally, if $n=q((k+1) d)+r$ with $q$ and $r$ integers and $0 \leqslant r<(k+1) \cdot d$, then subadditivity of $L$ gives $L(D: n) \leqslant q \cdot d+\min \{d, r\}$, and inspection of the subsequence

$$
\begin{aligned}
1, \ldots, d,(k+1) d+1, \ldots,(k+1) d+d, \ldots,(q-1)((k+1) d)+1, \ldots, \\
(q-1)((k+1) d)+d, q(k+1) d+1, \ldots, q(k+1) d+\min \{d, r\}
\end{aligned}
$$

shows $L(D: n) \geqslant q \cdot d+\min \{d, r\}$. Thus for all integers $n$,

$$
L(D: n)=d \cdot \llbracket \frac{n}{(k+1) \cdot d} \rrbracket+\min \{d, n \bmod [(k+1) d]\},
$$

which proves the theorem.

We can now answer Problem Three for the chess player problem. We have

$$
\begin{aligned}
L(\{21\}: 132) & =21 \cdot \llbracket \frac{132}{2 \cdot 21} \rrbracket+\min \{21,132(\bmod 42)\} \\
& =21 \cdot 3+\min \{21,6\}=69 .
\end{aligned}
$$

Thus the conclusion to Liu's chess player problem will still hold if 132 games are played in 70 days.

TheOREM 3.4. If $D=\{d, d+1,2 d+1\}$ with $d$ a positive integer, then

$$
L(D: n)=d \cdot \llbracket \frac{n}{3 d+1} \rrbracket+\min \{d, n \bmod [3 d+1]\}
$$

Proof. First we show that $L(D: 3 d+1)=d$. To this end suppose $a_{1}<\cdots<a_{t}$ is a subsequence of $1, \ldots, 3 d+1$ with the property that $\left|a_{j}-a_{i}\right| \notin D$ for $1 \leqslant i<j$ $\leqslant t$. From the sequence $\left\{a_{i}\right\}_{1}^{t}$ we define two more sequences $\left\{b_{i}\right\}_{1}^{t}$ and $\left\{c_{i}\right\}_{1}^{t}$ as follows.

If $a_{i} \leqslant d$, then $b_{i}=a_{i}+d+1$ and $c_{i}=a_{i}+2 d+1$.

If $d<a_{i} \leqslant 2 d+1$, then $b_{i}=a_{i}-d$ and $c_{i}=a_{i}+d+1$.

If $2 d+1<a_{i}$ then $b_{i}=a_{i}-d$ and $c_{i}=a_{i}-2 d-1$.

A careful examination of the defining relations for the sequences $\left\{b_{i}\right\}_{1}^{t}$ and $\left\{c_{i}\right\}_{1}^{t}$ shows that the sequences $\left\{a_{i}\right\}_{1}^{t},\left\{b_{i}\right\}_{1}^{t}$ and $\left\{c_{i}\right\}_{1}^{t}$ comprise $3 t$ distinct integers in the range $1, \ldots, 3 d+2$. Thus $3 t \leqslant 3 d+2$ so $t<d+1$. But $t \leqslant d$ implies $L(D: 3 d+1)$ $\leqslant d$. On the other hand, the sequence $a_{i}=i$ for $1 \leqslant i \leqslant d$ satisfies $\left|a_{i}-a_{j}\right| \notin D$ for $i \neq j$. Thus $L(D: 3 d+1)=d$. The remainder of the proof of Theorem 3.4 follows hv the same type of argument used to prove Theorem 3.3. 
We now use Theorem 3.4 to solve Problem Three for the case of the elevator problem (Problem Two). One has

$$
\begin{aligned}
L(\{9,9+1,2 \cdot 9+1\}: 100) & =9 \cdot \llbracket \frac{100}{28} \rrbracket+\min \{9,100(\bmod 28)\} \\
& =9 \cdot 3+\min \{9,16\}=36 .
\end{aligned}
$$

Thus, to assure the elevator stops at floors that are exactly 9,10 or 19 stories apart one needs to know the elevator made at least $36+1=37$ stops.

There are other special cases where conclusions like those of Theorems 3.3. and 3.4 can be obtained. For example, if $D$ has the form

$$
D=\{d, d+1,2 d+1,3 d+2, \ldots,(k+1) d+k\}
$$

for $d$ and $k$ positive integers, then one obtains

$$
L(D: n)=d \cdot \llbracket \frac{n}{(k+2) d+k} \rrbracket+\min \{d, n \bmod [(k+2) d+k]\} .
$$

A similar result obtains when $D$ is any set of consecutive integers or any set of consecutive multiplies of a fixed integer.

We note that in each of the special cases discussed so far there exist integers $M$ and $K$ such that

$$
L(D: n+M)=K+L(D: n) \text { for } n=1,2, \ldots
$$

This is not the case in general. For example, if $D$ is of the form $D=\{1, d, d+1\}$, the behavior of $L(D: n)$ is quite different. However, computer runs indicate that for every choice of $D$ there exist integers $N, M$ and $K$ such that

$$
L(D: n+M)=K+L(D: n) \text { whenever } n \geqslant N .
$$

This result will be proved in $\$ 4$.

4. Asymptotic behavior of $L(D: n)$. The main result of this section is

THEOREM 4.1. Let $D$ be any nonvoid finite set of positive integers. Then there exist integers $N, M$ and $K$ (which depend only on $D$ ) such that

$$
L(D: n+M)-L(D: n)=K \text { whenever } n \geqslant N \text {. }
$$

We postpone the proof of Theorem 4.1 until we have established some preliminary results. First we define the notion of the $L$-density, $\rho(D)$, of a set $D$ of positive integers.

Definition. Let $D$ be a finite nonvoid set of positive integers. Then the $L$-density of $D$ is defined to be

$$
\rho(D)=\lim _{n \rightarrow \infty} \frac{L(D: n)}{n},
$$

provided such a limit exists.

Note. given that Theorem 4.1 holds, we have $\rho(D)=K / M$ where $K$ and $M$ are as in the theorem. We establish upper and lower bounds for the ration $L(D: n) / n$. 
TheOREM 4.2. Let $D=\left\{d_{1}, \ldots, d_{k}\right\}$ where $0<d_{1}<\cdots<d_{k}$. Then $(1 /(k+1)) \leqslant$ $\lim \inf L(D: n) / n \leqslant \lim \sup L(D: n) / n \leqslant 1 / 2$. Moreover, $\quad(1 /(k+1)) \leqslant$ $L(D: n) / n$ for every positive integer $n$.

Proof. Note that $\left\{d_{1}\right\} \subseteq D$. Thus Theorem 3.1 (i) implies $L(D: n) \leqslant L\left(\left\{d_{1}\right\}: n\right)$ for all positive integers $n$. If we divide both sides of this inequality by $n$ and use Theorem 3.3 to take limits, we obtain

$$
\lim \sup \frac{L(D: n)}{n} \leqslant \lim d_{1} \cdot \llbracket \frac{n}{2 d_{1}} \rrbracket \cdot n^{-1}=\frac{1}{2} .
$$

To complete the proof we need only show $(1 /(k+1)) \leqslant L(D: n) / n$ for $n \geqslant 1$. To this end we utilize the "first choice" subsequence of $1, \ldots, n$ defined by $a_{1}=1$, and if $a_{1}, a_{2}, \ldots, a_{j}$ have been selected, then $a_{j+1}$ is the smallest integer less than or equal to $n$ such that $\left|a_{j+1}-a_{i}\right| \notin D$ for $1 \leqslant i \leqslant j$. Suppose this sequence consists of the $t$-terms $a_{1}, \ldots, a_{t}$. Since $\left\{a_{i}\right\}_{1}^{t}$ is the "first choice" sequence, each integer in $\{1, \ldots, n\}$ is either equal to some $a_{i}$ or is of the form $a_{i}+d_{l}$ for some $i, l$ with $1 \leqslant i \leqslant t$ and $1 \leqslant l \leqslant k$. Thus $n \leqslant(k+1) \cdot t$. But $t \leqslant L(D: n)$, so $n \leqslant(k+1) \cdot L(D: n)$, which completes the proof of Theorem 4.2.

Our proofs of Theorem 4.1 and of the lemmas used to establish it require some special terminology. Let $\left\{a_{i}\right\}_{1}^{t}$ be a sequence of integers with $t \geqslant 2$. We define the sequence of gaps associated with $\left\{a_{i}\right\}_{1}^{t}$ to be the sequence $\left\{g_{i}\right\}_{1}^{t-1}$ where $g_{i}=a_{i+1}-a_{i}$ for $i=1, \ldots, t-1$. Note that the sequence $\left\{a_{i}\right\}_{1}^{t}$ is uniquely determined by its first term $a_{1}$ and its gap sequence $\left\{g_{i}\right\}_{1}^{t-1}$. For the remainder of this paper $D=\left\{d_{1}, \ldots, d_{k}\right\}$ shall denote a fixed set of positive integers with $1 \leqslant d_{1}<\cdots<d_{k}$.

We choose $U$ to be the fixed upper bound $U=d_{k}+1$ of the set $D$. We say $\left\{a_{i}\right\}_{1}^{t}$ is admissible for $L(D: n)$ in case $1 \leqslant a_{1}<\cdots<a_{t} \leqslant n$ and $\left|a_{i}-a_{j}\right| \notin D$ whenever $i \neq j$. We say $\left\{a_{i}\right\}_{1}^{t}$ realizes $L(D: n)$ in case $\left\{a_{i}\right\}_{1}^{t}$ is admissible and $t=L(D: n)$. For example, both $1,2,3,7$ and $1,5,6,7$ realize $L(\{3\}: 7)$. If $\left\{g_{i}\right\}_{1}^{t-1}$ is a sequence of positive integers then the block $B=h_{1}, \ldots, h_{l}$ of index $j$ and length $l$ is said to appear in the sequence $\left\{g_{i}\right\}_{1}^{t-1}$ in case $h_{i}=g_{j+(i-1)}$ for $1 \leqslant i \leqslant l$. Two blocks $B=h_{1}, \ldots, h_{l}$ and $B^{\prime}=h_{1}^{\prime}, \ldots, h_{m}^{\prime}$ are called identical in case $l=m$ and $h_{i}=h_{i}^{\prime}$ for $1 \leqslant i \leqslant l$. We denote the length of the block $B$ as $l(B)$, and we denote the sum of the terms in the block $B$ as $\Sigma B$. The block density of the block $B$ is defined as the ratio $\delta(B)=l(B) / \Sigma B$. We shall order sequences (and blocks) of the same length by the lexicographical ordering. Thus $\left\{a_{i}\right\}_{1}^{t}<\left\{a_{i}^{\prime}\right\}_{1}^{t}$ in case there is an integer $m \leqslant t$ such that $a_{i}=a_{i}^{\prime}$ for $i<m$ and $a_{m}<a_{m}^{\prime}$.

We now give the preliminary results used to prove Theorem 4.1. Taken together, these results identify a repeating block structure in gap sequences. It is this repeating block structure which forces the periodic increase of $L(D: n)$ asserted in Theorem 4.1. Moreover, in Theorem 4.6 we use the block density $\delta(B)$ of such repeating blocks to compute $\rho(D)=\lim _{n \rightarrow \infty} L(D: n) / n$.

Lemma 4.3. Suppose $L(D: n)>L(D: n-1)$. Let $\left\{a_{i}\right\}_{1}^{t}$ be any realizing sequence for $L(D: n)$ and let $\left\{g_{i}\right\}_{1}^{t-1}$ be the associated gap sequence, viewed as a single block $B=g_{1}, \ldots, g_{t-1}$. Then $a_{1}=1, a_{t}=n, \Sigma B=n-1$ and $L(D: n)=l(B)+1$. 
Proof. If $a_{1}>1$ then the sequence $\left\{a_{i}-1\right\}_{1}^{t}$ would be an admissible sequence for $L(D: n-1)$. But this contradicts the assumption $L(D: n)>L(D: n-1)$, since $L(D: n-1)$ is greater than or equal to the length of any admissible sequence of $L(D: n-1)$. If $a_{t}<n$, then $\left\{a_{i}\right\}_{1}^{t}$ would be an admissible sequence for $L(D: n-1)$, so that $L(D: n-1) \geqslant t=L(D: n)$, again a contradiction. Therefore $a_{1}=1$ and $a_{t}=n$. Finally, by the definition of gap sequences, we have $t=L(D: n)$ $=l(B)+1$ and $\sum B=\sum_{i=1}^{t-1}\left(a_{i+1}-a_{i}\right)=a_{t}-a_{1}=n-1$. This proves the lemma.

Lemma 4.4. Suppose that $L(D: n)>L(D: n-1)$. Let $\left\{a_{i}\right\}_{1}^{t}$ be minimal in the lexicographical ordering among all sequences that realize $L(D: n)$. Let the associated gap sequence have block forms $I_{1}, A, B_{1}, C, T_{1}$ and $I_{2}, A, B_{2}, C, T_{2}$ where $l(A), l(C)$ $\geqslant d_{k}+1$. If $l\left(B_{1}\right)=l\left(B_{2}\right)$, then $B_{1}$ and $B_{2}$ are identical.

Proof. Let $\left\{a_{i}\right\}_{1}^{t}$ with associated gap sequence $\left\{g_{i}\right\}_{1}^{t-1}$ having block forms $I_{1}, A, B_{1}, C, T_{1}$ and $I_{2}, A, B_{2}, C, T_{2}$ be as in the statement of the lemma. By Lemma 4.3 we have

$$
\begin{aligned}
n & =1+\sum I_{1}+\sum A+\sum B_{1}+\sum C+\sum T_{1} \\
& =1+\sum I_{2}+\sum A+\sum B_{2}+\sum C+\sum T_{2} .
\end{aligned}
$$

If, say $\sum B_{2}<\sum B_{1}$, we could define a new subsequence $\left\{a_{i}^{\prime}\right\}_{1}^{t}$ of $1, \ldots, n$ by $a_{1}^{\prime}=a_{1}=1$ and $\left\{a_{i}^{\prime}\right\}_{1}^{t}$ has $I_{1}, A, B_{2}, C, T_{1}$ as gap sequence. Since $l(A), l(B) \geqslant U$, the sequence $\left\{a_{i}^{\prime}\right\}_{1}^{t}$ is admissible for $L\left(D: 1+\sum I_{1}+\sum A+\sum B_{2}+\Sigma C+\Sigma T_{1}\right)$, contrary to the assumption $L(D: n)>L(D: n-1)$, and the fact that $L$ is a nondecreasing function of its integer argument. Thus, $\sum B_{1} \leqslant \Sigma B_{2}$. Likewise, $\Sigma B_{2} \leqslant$ $\sum B_{1}$, so $\sum B_{1}=\sum B_{2}$.

If $B_{1}$ and $B_{2}$ are not identical, then one of them, say $B_{2}$, is smaller in the lexicographical ordering. If $B_{2}<B_{1}$, consider the sequence $\left\{a_{i}^{\prime}\right\}_{1}^{t}$ defined by $a_{1}^{\prime}=a_{1}$ and the gap sequence $I_{1}, A, B_{2}, C, T_{1}$. This sequence both realizes $L(D: n)$ and precedes $\left\{a_{i}\right\}_{1}^{t}$ lexicographically, contrary to the assumption of $\left\{a_{i}\right\}_{1}^{t}$ being minimal among realizing sequences. Thus $B_{2} \geqslant B_{1}$. Likewise, $B_{1} \geqslant B_{2}$, which proves the lemma.

LEMMA 4.5. Let $U=d_{k}+1$ and suppose $L(D: n)>3 U^{2 U}$ where $L(D: n)>$ $L(D: n-1)$. Let $\left\{a_{i}\right\}_{1}^{t}$ be minimal in the lexicographical ordering among all sequences realizing $L(D: n)$. Then there exist an integer $p \leqslant U^{2 U}$ and blocks $I, B$ and $T$, each of length $\leqslant p$, such that the gap sequence associated with $\left\{a_{i}\right\}_{1}^{t}$ has block form $I, B, \ldots, B, \tilde{B}, T$ where $\tilde{B}$ is an initial segment of the block $B$ or $\tilde{B}$ is the null block.

Proof. Let $\left\{g_{i}\right\}_{1}^{t-1}$ be the gap sequence associated to the lexicographically minimal sequence realizing $L(D: n)$. Let $z=L(D: n)-\left(U^{2 U}+U+1\right)$ and consider the $U^{2 U}+1$ pairs of blocks $P_{m}=\left(\left\{g_{i}\right\}_{m}^{m+U-1},\left\{g_{i}\right\}_{m+z}^{m+U-1+z}\right)$ where $1 \leqslant m \leqslant$ $U^{2 U}+1$. Since each $g_{i} \leqslant d_{k}+1=U$, there are at most $U^{2 U}$ such distinct choices for the pairs $P_{m}$. Hence, by the pigeonhole principle, there exist integers $m_{1}$ and $m_{2}$ with $1 \leqslant m_{1}<m_{2} \leqslant U^{2 U}+1$ and $P_{m_{1}}=\left(A_{1}, C_{1}\right)$ identical to $P_{m_{2}}=\left(A_{2}, C_{2}\right)$. Since $l\left(A_{1}\right)=l\left(C_{1}\right)=U$, Lemma 4.4 applies, so that $\left\{g_{i}\right\}_{m_{1}}^{m_{1}+U-1+z}$ and $\left\{g_{i}\right\}_{m_{2}}^{m_{2}+U-1+z}$ are identical gap sequences. Take $B$ to be the block $g_{m_{1}}, g_{m_{1}+1}, \ldots, g_{m_{2}-1}$. Note $l(B)=$ $m_{2}-m_{1} \leqslant U^{2 U}$. Since $g_{m_{1}+i}=g_{m_{2}+i}$ for $0 \leqslant i \leqslant U-1+z$, it follows that 
$\left\{g_{i}\right\}_{m_{1}}^{m_{2}}+U-1+z$ consists of repeated copies of the block $B$ followed, perhaps, by an initial segment $\tilde{B}$ of the block $B$ (depending on whether or not $l(B)$ exactly divides $U+z)$. Let $I=g_{1}, \ldots, g_{m_{1}-1}$ be the initial block of length $l(I)=m_{1}-1<U^{2 U}$, and let $T=g_{m_{2}+U+z}, \ldots, g_{t-1}$ be the terminal block of length

$$
l(T)=L(D: n)-\left(m_{2}+U+z\right)=U^{2 U}+1-m_{2}<U^{2 U} .
$$

If we take $p$ to be the maximum of $l(I), l(B)$ and $l(T)$, then $p, I, B$ and $T$ satisfy $l(I), l(B), l(T) \leqslant p \leqslant U^{2 U}$ with $I, B, \ldots, B, \tilde{B}, T$ being the block form of the gap sequence $\left\{g_{i}\right\}_{1}^{t-1}$. This completes the proof of Lemma 4.5 .

According to Lemma 4.5, if $n$ is sufficiently large and $L(D: n)>L(D: n-1)$, then the gap sequence associated with the lexicographically minimal realizing sequence for $L(D: n)$ has the form $I, B, \ldots, B, T$ where, if necessary, the block $\tilde{B}$ has been absorbed into $T$. Each such block $I, B$ and $T$ has bounded length, the bound being independent of $n$. In this setting we say the triple $\langle I, B, T\rangle$ realizes $L(D: n)$. Let \& $^{\prime}$ be the set of all triples $\langle I, B, T\rangle$ which realize $L(D: n)$ at infinitely many values of $n$ for which $L(D: n)>L(D: n-1)$. Note that the set $\mathfrak{B}$ is finite. Let $K$ be the least common multiple of the lengths of all blocks $B_{\alpha}$ where $\left\langle I_{\alpha}, B_{\alpha}, T_{\alpha}\right\rangle \in \mathcal{H}$. Each realization of the minimal gap sequence for $\left\langle I_{\alpha}, B_{\alpha}, T_{\alpha}\right\rangle \in H^{4}$ can be expressed as $\bar{I}_{\alpha}, \bar{B}_{\alpha}, \ldots, \bar{B}_{\alpha}, \bar{T}_{\alpha}$ where $\bar{I}_{\alpha}=I_{\alpha}, \bar{B}_{\alpha}$ is $K / l\left(B_{\alpha}\right)$ repetitions of $B_{\alpha}$, and where $\bar{T}_{\alpha}$ is that segment of the original representation $I_{\alpha}, B_{\alpha}, \ldots, B_{\alpha}, T_{\alpha}$ which cannot be absorbed in the last copy of $\bar{B}_{\alpha}$. By Lemma 4.5 the lengths of all such blocks $\bar{I}_{\alpha}, \bar{B}_{\alpha}$ and $\bar{T}_{\alpha}$ are bounded independently of $n$ and $\alpha$. Let $\mathfrak{B} / K$ be the collection of all triples $\left\langle\bar{I}_{\alpha}, \bar{B}_{\alpha}, \bar{T}_{\alpha}\right\rangle$ obtained in this manner. We $\operatorname{read} \mathfrak{R} / K$ as “ $B \bmod K$ ". Note if $\left\langle\bar{I}_{\alpha}, \bar{B}_{\alpha}, \bar{T}_{\alpha}\right\rangle \in \mathfrak{B} / K$ then $l\left(\bar{B}_{\alpha}\right)=K$. Our next result utilizes the set $\mathfrak{H} / K$ to show $\rho(D)$ exists and is given by $\rho(D)=\delta(B)$ for any block $B$ for which $\langle I, B, T\rangle \in$ *

THEOREM 4.6. Let $\left\langle I_{\alpha}, B_{\alpha}, T_{\alpha}\right\rangle$ and $\left\langle I_{\beta}, B_{\beta}, T_{\beta}\right\rangle$ be triples in 18 with $\left\langle\bar{I}_{\alpha}, \bar{B}_{\alpha}, \bar{T}_{\alpha}\right\rangle$ and $\left\langle\bar{I}_{\beta}, \bar{B}_{\beta}, \bar{T}_{\beta}\right\rangle$ corresponding triples in $\mathfrak{B} / K$. Then $\sum \bar{B}_{\alpha}=\sum \bar{B}_{\beta}$ and $\delta\left(B_{\alpha}\right)=\delta\left(B_{\beta}\right)$ $=\delta\left(B_{\alpha}\right)=\rho(D)$.

Proof (Notation as above). The integer $K$ is the least common multiple of the lengths $l\left(B_{\alpha}\right)$. Each $\bar{B}_{\alpha}$ consists of $K / l\left(B_{\alpha}\right)$ copies of $B_{\alpha}$, so $l\left(\bar{B}_{\alpha}\right)=K$ for all $\alpha$. Suppose $\sum \bar{B}_{\beta}<\sum \bar{B}_{\alpha}$ for two triples $\left\langle\bar{I}_{\alpha}, \bar{B}_{\alpha}, \bar{T}_{\alpha}\right\rangle$ and $\left\langle\bar{I}_{\beta}, \bar{B}_{\beta}, \bar{T}_{\beta}\right\rangle$ in $\mathfrak{B} / K$. Then $\sum \bar{B}_{\alpha}=r+\sum \bar{B}_{\beta}$ for some positive integer $r$. Pick $s$ to be a positive integer so that $s \cdot l\left(\bar{B}_{\beta}\right)>l\left(\bar{I}_{\alpha}\right)+l\left(\bar{T}_{\alpha}\right)$. We can choose $k$ large enough so that $k \cdot r+\sum \bar{I}_{\alpha}+\sum \bar{T}_{\alpha}$ $>s \cdot \sum \bar{B}_{\beta}$. Then, since $\left\langle\bar{I}_{\alpha}, \bar{B}_{\alpha}, \bar{T}_{\alpha}\right\rangle \in \mathfrak{B} / K$ we can also choose $k$ so that

$$
L\left(D: 1+\sum \bar{I}_{\alpha}+k \cdot \sum \bar{B}_{\alpha}+\sum \bar{T}_{\alpha}\right)=1+l\left(\bar{I}_{\alpha}\right)+k \cdot l\left(\bar{B}_{\alpha}\right)+l\left(\bar{T}_{\alpha}\right) .
$$

Thus

$$
\begin{aligned}
L(D: 1 & \left.+\sum \bar{I}_{\alpha}+k \cdot \sum \bar{B}_{\alpha}+\sum \bar{T}_{\alpha}\right)=L\left(D: 1+\sum \bar{I}_{\alpha}+k \cdot\left(r+\sum \bar{B}_{\beta}\right)+\sum \bar{T}_{\alpha}\right) \\
& =L\left(D: 1+k \cdot r+\sum \bar{I}_{\alpha}+\sum \bar{T}_{\alpha}+k \cdot \sum \bar{B}_{\beta}\right) \geqslant L\left(D: 1+(s+k) \cdot \sum \bar{B}_{\beta}\right) \\
& \geqslant 1+(s+k) \cdot l\left(\bar{B}_{\beta}\right)=1+k \cdot l\left(\bar{B}_{\beta}\right)+s \cdot l\left(\bar{B}_{\beta}\right) \\
& >1+l\left(\bar{I}_{\alpha}\right)+k \cdot l\left(\bar{B}_{\beta}\right)+l\left(\bar{T}_{\alpha}\right)=L\left(D: 1+\sum \bar{I}_{\alpha}+k \cdot \sum \bar{B}_{\alpha}+\sum \bar{T}_{\alpha}\right),
\end{aligned}
$$


which is a contradiction. Likewise $\sum \bar{B}_{\alpha}<\sum \bar{B}_{\beta}$ is impossible, so $\sum \bar{B}_{\alpha}=\sum \bar{B}_{\beta}$ is constant. Let $M=\sum \bar{B}_{\alpha}$ denote this constant.

Now, suppose $\bar{B}_{\alpha}$ consists of $q$ copies of $B_{\alpha}$. Then

$$
\delta\left(\bar{B}_{\alpha}\right)=l\left(\bar{B}_{\alpha}\right) / \sum \bar{B}_{\alpha}=\left[q \cdot l\left(B_{\alpha}\right)\right] /\left[q \cdot \sum B_{\alpha}\right]=K / M=\delta\left(B_{\alpha}\right)
$$

is also constant. Thus $\delta\left(\bar{B}_{\alpha}\right)=\delta\left(B_{\alpha}\right)=\delta\left(B_{\beta}\right)$. Finally, by Lemma 4.5 , if $n>3 U^{2 U}$ we have $L$ increases only at integers of the form $1+l\left(\bar{I}_{\alpha}\right)+k \cdot l\left(\bar{B}_{\alpha}\right)+l\left(\bar{T}_{\alpha}\right)$ for some $\left\langle\bar{I}_{\alpha}, \bar{B}_{\alpha}, \bar{T}_{\alpha}\right\rangle$ in $\mathfrak{B} / K$. Let $l_{\max }, l_{\min }, \Sigma_{\max }, \Sigma_{\min }$ denote, respectively, the maximum and minimum of $1+l\left(\bar{I}_{\alpha}\right)+l\left(\bar{T}_{\alpha}\right)$ and $1+\sum \bar{I}_{\alpha}+\sum \bar{T}_{\alpha}$ for $\left\langle\bar{I}_{\alpha}, \bar{B}_{\alpha}, \bar{T}_{\alpha}\right\rangle \in \mathfrak{B} / K$. Then for large $n$ we have $L(D: n)=1+l\left(\bar{I}_{\alpha}\right)+k \cdot l\left(\bar{B}_{\alpha}\right)+$ $l\left(\bar{T}_{\alpha}\right)$ for some integer $k$ and some $\left\langle\bar{I}_{\alpha}, \bar{B}_{\alpha}, \bar{T}_{\alpha}\right\rangle \in \mathfrak{B} / K$. Thus

$$
\frac{k \cdot l\left(\bar{B}_{\alpha}\right)+l_{\min }}{(k+1) \cdot \sum \bar{B}_{\alpha}+\sum_{\max }} \leqslant \frac{L(D: n)}{n} \leqslant \frac{(k+1) l\left(\bar{B}_{\alpha}\right)+l_{\max }}{k \cdot \sum \bar{B}_{\alpha}+\sum_{\min }} .
$$

Noting that $l\left(\bar{B}_{\alpha}\right)=K$ and $\sum \bar{B}_{\alpha}=M$ are constants, we can take limits to establish $\delta\left(\dot{B_{\alpha}}\right)=\rho(D)$, which proves Theorem 4.6.

We now give the proof of Theorem 4.1, that there exist integers $M, K$ and $N$ such that $L(D: n+M)-L(D: n)=K$ whenever $n \geqslant N$.

Let $M=\sum \bar{B}_{\alpha}$ for $\left\langle\bar{I}_{\alpha}, \bar{B}_{\alpha}, \bar{T}_{\alpha}\right\rangle \in \mathfrak{B} / K$ and let $K=l\left(\bar{B}_{\alpha}\right)$. Choose $N$ to be the least integer such that if $n \geqslant N$ and $L$ increases at $n$, then the gap sequence $\bar{I}_{\alpha}, \bar{B}_{\alpha}, \ldots, \bar{B}_{\alpha}, \bar{T}_{\alpha}$ associated with the triple $\left\langle\bar{I}_{\alpha}, \bar{B}_{\alpha}, \bar{T}_{\alpha}\right\rangle$ in $\mathfrak{B} / K$ realizing $L(D: n)$ has at least two copies of the block $\bar{B}_{\alpha}$. Thus we can choose $N$ so that $N \geqslant 2 \cdot K+$ $\Sigma_{\max }$.

Our next result shows $L(D: n+M)=L(D: n)+K$ whenever $n \geqslant N$ and $L$ increases at $n$.

Claim. If $n \geqslant N$ and $L(D: n)>L(D: n-1)$ with $L(D: n)$ realized by $\left\langle\bar{I}_{\alpha}, \bar{B}_{\alpha}, \bar{T}_{\alpha}\right\rangle$ in $\mathfrak{B} / K$, then $L(D: n+M)>L(D: n+M-1)$, and $L(D: n+M)$ is also realized by $\left\langle\bar{I}_{\alpha}, \bar{B}_{\alpha}, \bar{T}_{\alpha}\right\rangle$ with the realization of $L(D: n+M)$ requiring one more copy of $\bar{B}_{\alpha}$ than the realization of $L(D: n)$. Thus $L(D: n+M)=L(D: n)+$ $K$ if $n \geqslant N$ and $L$ increases at $n$.

Proof. Let $n>N$ where $L(D: n)>L(D: m)$ for $m<n$ and let $L(D: n)$ be realized by $\left\langle\bar{I}_{\alpha}, \bar{B}_{\alpha}, \bar{T}_{\alpha}\right\rangle \in \mathfrak{B} / K$ with the corresponding gap sequence consisting of $\bar{I}_{\alpha}, r$-copies of $\bar{B}_{\alpha}$, followed by $\bar{T}_{\alpha}$. If $L(D: n+M-1)=L(D: n+M)$, choose $m^{*}$ to be the largest integer less than $n+M$ such that $L$ increases at $m^{*}$. Since the sequence $\left\{a_{i}\right\}_{1}^{L(D: n)+K}$ defined by $a_{1}=1$ and the gap sequence $\bar{I}_{\alpha},(r+1)$-copies of $\bar{B}_{\alpha}$, followed by $\bar{T}_{\alpha}$ is admissible for $L(D: n+M)$, we have

$$
L\left(D: m^{*}\right)=L(D: n+M) \geqslant L(D: n)+K .
$$

Thus $m^{*}>n>N$. Let $\left\langle\bar{I}_{\beta}, \bar{B}_{\beta}, \bar{T}_{\beta}\right\rangle$ in $\mathscr{B} / K$ realize $L\left(D: m^{*}\right)$ with the actual realization consisting of $\bar{I}_{\beta}$,s-copies of $\bar{B}_{\beta}$, followed by $\bar{T}_{\beta}$. Since $m^{*}>N$ we have $s \geqslant 2$. But then the sequence $\left\{a_{i}^{\prime}\right\}$ defined by $a_{1}^{\prime}=1$ and the gap sequence $\bar{I}_{\beta}$, $(s-1)$-copies of $\bar{B}_{\beta}$, followed by $\bar{T}_{\beta}$ is admissible ${ }^{1}$ for $L\left(D: m^{*}-M\right)$. Thus

$$
L\left(D: m^{*}-M\right) \geqslant 1+l\left(\bar{I}_{\beta}\right)+(s-1) \cdot K+l\left(\bar{T}_{\beta}\right) .
$$

\footnotetext{
${ }^{1}$ This assertion assumes $K>U$, which is no loss of generality.
} 
But $L\left(D: m^{*}\right) \geqslant L(D: n)+K$ implies

$$
1+l\left(\bar{I}_{\beta}\right)+(s-1) K+l\left(\bar{T}_{\beta}\right) \geqslant 1+l\left(\bar{I}_{\alpha}\right)+r \cdot K+l\left(\bar{T}_{\alpha}\right)=L(D: n) .
$$

Thus $L\left(D: m^{*}-M\right) \geqslant L(D: n)$, which contradicts the assumption that $L$ increases at $n$, since $m^{*}-M<n$. Thus $L(D: n+M)>L(D: n+M-1)$.

Since $L$ increases at $n+M$ we have $L(D: n+M)$ is realized by some triple $\left\langle\bar{I}_{\beta}, \bar{B}_{\beta}, \bar{T}_{\beta}\right\rangle$ in $\mathfrak{B} / K$. We have shown that the sequence $\left\{a_{i}\right\}_{1}^{L(D: n)+K}$ defined by $a_{1}=1$ and the gap sequence $\bar{I}_{\alpha},(r+1)$-copies of $\bar{B}_{\alpha}$, followed by $\bar{T}_{\alpha}$ is admissible for $L(D: n+M)$. If some other triple $\left\langle\bar{I}_{\beta}, \bar{B}_{\beta}, \bar{T}_{\beta}\right\rangle$ in $\mathscr{B} / K$ realized $L(D: n+M)$, say with $s$-copies of $\bar{B}_{\beta}$, then, since $n+M>N$, we would have $s \geqslant 2$. But then the corresponding gap sequence $\bar{I}_{\beta}$, followed by $(s-1)$-copies of $\bar{B}_{\beta}$, followed by $\bar{T}_{\beta}$ would be admissible for $L(D: n)$, would have the same length $l\left(\bar{I}_{\beta}\right)+(s-1) K+$ $l\left(\bar{T}_{\beta}\right)$ and would precede $\bar{I}_{\alpha}, \bar{B}_{\alpha}, \ldots, \bar{B}_{\alpha}, \bar{T}_{\alpha}$ in the lexicographical ordering. This contradicts $\left\langle\bar{I}_{\alpha}, \bar{B}_{\alpha}, \bar{T}_{\alpha}\right\rangle$ realizing $L(D: n)$, which establishes the claim.

We can now complete the proof of Theorem 4.1. For $n \geqslant N$, let $n^{*}$ be the largest integer less than or equal to $n$ such that $L$ increases at $n^{*}$. Then by the claim we have $L\left(D: n^{*}+M\right)=L(D: n)+K$. Note $L(D: n+M) \geqslant L\left(D: n^{*}+M\right)$. If $L(D: n+M)>L\left(D: n^{*}+M\right)$, let $n^{* *}$ be the smallest integer less than or equal to $n+M$ for which $L\left(D: n^{* *}\right)=L(D: n+M)$. Then the same argument used in proving the claim shows $n^{*}<n^{* *}-M<n$ with $L$ increasing at $n^{* *}-M$, contrary to the choice of $n^{*}$. Thus $L(D: n+M)=L(D: n)+K$ for all $n \geqslant N$, which proves Theorem 4.1.

\section{REFERENCES}

1. Michael Gilpin and Robert Shelton, Problem 1152, Math. Mag. 55 (1982), 237.

2. C. L. Liu, Elements of discrete mathematics, McGraw-Hill, New York, 1977.

3. __ Topics in combinatorial mathematics, Math. Assoc. Amer., Washington, D.C., 1972.

Department of Mathematical and Computer Sciences, Michigan Technological University, Houghton, Michigan 49931 